\title{
On the limits of Kagan's impulsive reflective distinction
}

\author{
BILL JONES \\ Carleton University, Ottawa, Ontario, Canada \\ and \\ LESLIE MCINTYRE \\ University of Queensland, St. Lucia, Queensland 4067, Australia
}

\begin{abstract}
A logical analysis is made of the Matching Familiar Figures (MFF) Test on the basis of which children have been classified as "impulsive" or "reflective." The reflective strategy is implicitly preferred to the impulsive because the reflective child makes fewer errors though generally taking longer to make his first response. We show that the test allows the choice of a number of "game plans" and speed-accuracy tradeoffs which in practice may not be very different. Error rates may not indicate perceptual sensitivity, in any case, since sensitivity and response factors may be confounded in the error rate. Using a visual running-memory-span task to avoid the inherent difficulties of the MFF test, we found that children previousiy classified on the basis of that test as impulsive or reflective did not differ in recognition accuracy but did differ in response bias and response latency. Accuracy and bias are estimated by way of Luce's choice theory (Luce, 1963), and the results are discussed in those terms.
\end{abstract}

In the main, cognitive psychology has tended to avoid problems of individual differences dealing in models of memory and perceptual processes common to all adults or to all developing children, For this reason. Kagan's impulsive-reflective distinction emphasizing, as it does, differences in cognitive styles in children should be of special interest to cognitive psychologists. More particularly, the construct connects discriminative sensitivity and speed of judgment, a problem with which psychophysicists have recently been concerned (e.g., Pike, 1973). Our purpose here is to analyze the meaning and the general applicability of Kagan's distinction.

Briefly, Kagan (e.g., Kagan, 1966a, b; Kagan, Rosman, Day, Albert, \& Philips, 1964) has argued that perceptual recognition in children follows either an "impulsive" or a "reflective" strategy. Operationally, impulsive compared to reflective performance is associated with briefer average latencies and comparatively more errors on Kagan's Matching Familiar Figure Test (MFF). The MFF requires the child to choose the correct match for a line drawing from a 2 by 3 matrix of similar comparison items where five items are incorrect. If the child is in error, he makes a further choice until the correct item is located. Two response parameters are used: the mean (or total) latency for the first response to each item, and the total number of errors across all items (typically 12 or 24). Kagan (1966a) suggests that impulsivity and reflectivity are general cognitive styles

Address for reprints: Bill Jones, Department of Psychology, Carleton University, Ottawa, Ontario, Canada. related to general intellectual abilities and probably based upon constitutional predispositions. However, we wish to advance two broad reasons for thinking that the two groups of children may not, in fact, be ordinarily different in perceptual sensitivity, but that they may indeed differ in how they see the information processing demands of the MFF.

Our first point is that the MFF allows the child a wide latitude in the choice of "game plans." For example, when the child makes his first choice, the odds are only one in six of being correct. By quickly eliminating some alternatives against feedback from the experimenter, the child shortens the odds in his favor. Such a strategy would be a rational response if the child perceived the situation as one of obtaining positive knowledge of results in a fairly short time. However, describing the child as impulsive simply denies the rationality of his strategy. Kagan et al. (1964) have, in fact, suggested that "extreme impulsivity" may be one of the consequences of early brain damage. giving the impression that impulsivity is an abnormal piece of behavior which the child cannot control. Or, if not constitutionally predisposed, the impulsive child has low standards of performance (Kagan et al., 1964). In no discussion of impulsivity-reflectivity that we have seen has it been suggested that the impulsive child may be adopting a rational speed-accuracy tradeoff. If it is the case that the two groups of children "play the game" in a different way, we might learn from the MFF less than we originally suspected about discriminative sensitivity.

Our second argument against the utility of Kagan's 
paradigm concerns the operation of response biases. The MFF test is, in principle, a many alternative forced choice task in which probably not all items in the comparison set are equally discriminable and in which the individual child may be differentially biased toward some subset of the matrix. Such a bias toward (say) the top rather than the bottom half of the matrix would lead to the discriminability of the top items being overestimated and to the discriminability of the bottom items' being underestimated [both Ingleby (1974) and McNicol (1972) provide discussions of many alternative forced choice tasks]. Given a bias toward some items in the comparison matrix, the child will initialiy ignore others and generally tend to make more errors than the unbiased child, though not necessarily because of any inferior ability to discriminate alternatives. The discussion of impulsivityreflectivity by Kagan and others does, however, imply that a higher error rate of itself indicates lower discriminative sensitivity.

The general problem of assessing the value of the "impulsivity-reflectivity" construct is, therefore, that of obtaining measures of discriminative sensitivity which are relatively independent of the implicit cost and payoffs associated with the particular experimental test-independent, that is, of response biases, speed-accuracy strategies, and general "game plans." Ordinarily, the experimental methods derived from the theory of signal detection (see. e.g.. McNicol. 1972) provide ready ways of estimating sensitivity independent of response factors. However. signal detection methods usually require lengthy testing and the subject must be able to optimize response biases fairly systematically or else use a stimulus rating scale. Because children find such procedures quite difticult, Luce's choice theory (Luce. $1963)$ is an appropriate and generally analogous model which provides simple ways of independently estimating sensitivity and response bias (see also Ingleby, 1974, and McNicol, 1972). Since we intended to use a recognition memory paradigm, choice theory measures, in being based upon yes-no rather than rating scale data, have some further advantages. There is some evidence that signal detection analysis based on rating scales of recognition memory data may lead to artifactual findings (e.g., Gardner, MacFee. \& Krinsky, 1975). Although, in principle. it is possible to estimate parametric signal detection indices of recognition accuracy $\left(d^{\prime}\right)$ and response bias $(\beta)$ directly from tables, the use of those parameters requires us to meet some very strict assumptions about the nature of underlying "signal and noise" distributions, assumptions which it is practically impossible to test without rating scale data (see McNicol. 1972). Choice theory, on the other hand. requires no such assumptions.

Formally, the many alternative forced choice task with unequal discriminabilities and unequal biases can be handled by choice theory (such tasks become inordinately complex in signal detection terms: Ingleby. 1974; McNicol, 1972). We decided, however. to use another experimental paradigm for the following reasons. In practice, the problem of differential biases may be much more intractable than we have assumed for the purpose of exposition. Also, the MFF may not allow the collection within a comparatively short time of sufficiently stable data for choice-theory analysis, particularly if there are bias differences between reflective and impulsive groups. and, most importantly, it may not allow accurate specification of the latency distribution for each child. Typically, mean latency for each "impulsive" and "retlective" child has been estimated on the basis of 12-24 responses. Within-subject standard deviations are seldom, if ever, quoted, though it is a priori obvious that longer mean latencies would be obtained for the reflective group if those children made the occasional very long response.

Finally. and perhaps most importantly, we wished to test the applicability of Kagan's conceptual tempo notion to cognitive functions in general. In particular, we are concerned with short-term recognition memory since most models of discriminative sensitivity would include a short-term memory component. It is important to recognize that Kagan has stressed the general value of the reflective-impulsive construct. He talks about "a generalized behavioral tendency to be impulsive (or reflective) in problem situations where the child should consider the validity of his answers" (1966b, p. 24). Specifically, Kagan (1966b) and Siegel, Kirasic, and Kilburg (1973) have studied short-term recall or recognition in children described as impulsive or reflective on the basis of the MFF and have shown that, in general, impulsive children make somewhat more errors. In the present experiment, we obtained separate estimates of memory accuracy, response bias, and latency for a visual "running memory span" task (e.g., Shepard \& Teghtsoonian, 1961). The subject is shown, one at a time, a sequence of stimuli. In the present experiment, these were line drawings of common objects. Each picture appeared twice in the sequence and the subject's task was to say whether or not the item was appearing for the first time. Although the line drawings used in the present study were almost certainly easier to discriminate than typical MFF items. Siegel et al. (1973) have found that impulsive children tend to make more short-term recognition errors even given easily discriminable items which could be differentiated by name the difference between a pumpkin and a rabbit, for example), i.e., with material similar to our own.

Unlike the MFF, the running memory span allows little choice of "game plans." The child can make only one response for each test item, so that making the response will not delimit other alternatives. The operation of response biases is also much more simple 
to control. Finally, by allowing both recognition accuracy and latency to vary, our task makes possible an important test of the general usefulness of the impulsive-reflective distinction.

\section{METHOD}

\section{Subjects}

The subjects were 10 children who had previously been classified on the basis of the MFF, 5 as impulsive and 5 as reflective. Table 1 shows total error rates and mean first response times for both groups. The mean age of the impulsive group was 8 years 0 months (range 7 years 4 months to 8 years 7 months). The mean age of the retlective group was 8 years 1 month (range 7 years 4 months to 8 years 10 months). There were 3 boys and 2 girls in the impulsive group and 4 boys and 1 girl in the reflective group. All the subjects attended the same school in a predominantly working class area of Brisbane. Both groups were selected as the only children unequivocally impulsive or reflective in a random sample drawn for a larger study by the second author.

\section{Stimulus Preparation}

The stimuli were 68 slide photographs of drawings of common objects taken from the 1,000 most common words in the Thorndike-Lorge count. Each object was sketched by a professional illustrator using black ink on a white background, and two slides were made from each drawing.

\section{Apparatus}

The slides were shown on a screen using a Kodak Carousel. projector. Stimulus onset triggered a millisecond timer which was stopped by the child's vocal response. The response was picked up using a throat microphone and fed to the timer through a preamplifier.

\section{Procedure}

The children were tested individually in a quiet room in the school. The child sat about $1 \mathrm{~m}$ from the screen to one side of the projector. At that distance, the projected size of each slide on the screen was approximately $45 \mathrm{~cm}^{2}$. The experimenter explained to the child that he was about to see a sequence of pictures and that each picture would occur twice. He was to respond to each picture by saying "new" when any picture was shown for the first time, and "old" when the picture was shown for the second time. The throat microphone was placed comfortably around the child's neck, and he was told that the microphone would pick up his voice and stop the timer. Following a "ready" signal, each picture was presented at a shutter speed of $100 \mathrm{msec}$ and the child made his response. Sixteen practice trials (8 "old" and 8 "new" items) were used to familiarize the child with the procedure, followed by 120 experimental trials (60 "old" and 60 "new" items) with fresh stimuli. In all, the procedure took no more than $20 \mathrm{~min}$ on the average.

Table 1

Means and Standard Deviations for Total Error Scores (Emor) and Mean First Response Times in Seconds (Latency) Over 12 Items of the MFF Test for Five Implusive and Five Reflective Subjects

\begin{tabular}{lcccc}
\hline & \multicolumn{2}{c}{ Error } & \multicolumn{2}{c}{ Latency } \\
Subjects & Mean & SD & Mean & SD \\
\hline Impulsive & 19.8 & 2.23 & 5.86 & 1.87 \\
Reflective & 12.2 & 2.79 & 16.18 & 4.28 \\
\hline
\end{tabular}

Table 2

Means and Standard Deviations for Hit Rates (H), False Alarm Rates (FA), and the Derived Measures of Recognition Accuracy ( $\eta$ ) and Response Bias (b) for Each Subject Group

\begin{tabular}{|c|c|c|c|c|}
\hline & \multicolumn{2}{|c|}{ Impulsive } & \multicolumn{2}{|c|}{ Reflective } \\
\hline & Mean & SD & Mean & SD \\
\hline $\mathrm{H}$ & .792 & .26 & .892 & .12 \\
\hline FA & .046 & .05 & .126 & .08 \\
\hline$\eta$ & .114 & .13 & .140 & .14 \\
\hline b & 2.72 & 1.54 & .820 & .37 \\
\hline
\end{tabular}

\section{RESULTS}

\section{Probabilities}

Conventionally, we can describe two kinds of correct responses, the hit rate (the proportion of responses "old" given that the item was old) and the correct rejection rate (the proportion of responses "new" given that item was new). Correspondingly, there are two kinds of error, false alarms (the proportion of responses "old" given that the item was new) and the miss rate (the proportion of responses "new" when the item was old). According to choice theory, the subject's discriminative sensitivity or recognition accuracy, called $\eta$ (eta), is given by

$$
\eta=\left(\frac{\text { Misses } x \text { False Alarms }}{\text { Hits x Correct Rejections }}\right)^{1 / 2} \text {. }
$$

In general, $\eta$ will tend to zero as sensitivity increases. The subject's response bias, called $b$, is given by

$$
b=\left(\frac{\text { Misses } \times \text { Correct Rejections }}{\text { Hits } \times \text { False Alarms }}\right)^{1 / 2} \text {. }
$$

For unbiased subjects, $b$ will equal 1.0. In the present case, $b<1.0$ is associated with a bias toward saying "new." Table 2 gives means and standard deviations for hits, false alarms, $\eta$, and $b$.

Though the reflective group tend to have higher hit rates, the two groups did not differ significantly, $t(8)$ $=0.70$. The groups were significantly different in false-alarm rates, $\mathrm{t}(8)=1.98, \mathrm{p}<.05$. Converting the raw probabilities to $\eta$ and $b$ showed that impulsive and reflective groups did not differ significantly in recognition accuracy, $t(8)=.27$, but did differ significantly in response bias, $\mathrm{t}(8)=2.44, \mathrm{p}<.025$, the reflective group being on average somewhat biased to say "old" and the impulsive group rather more biased to say "new."

\section{Latencies}

For each subject, we calculated the mean latency (L) and the within-subject standard deviation around 
Table 3

Means and Standard Deviations for Average Latency (L), Latency Variance $\left(\sigma_{L}\right)$, Average Latency for Correct Responses $\left(\mathrm{L}_{\mathrm{CR}}\right)$, Latency Variance for Correct Responses $\left(\sigma_{\mathrm{LCR}}\right)$, Average Latency for Errors ( $L_{E R R}$ ), and Latency Variance Errors $\left(\sigma_{\text {LERR }}\right)$ for Both Subject Groups

\begin{tabular}{|c|c|c|c|c|}
\hline & \multicolumn{2}{|c|}{ Impulsive } & \multicolumn{2}{|c|}{ Reflective } \\
\hline & Mean & SD & Mean & SD \\
\hline $\mathrm{L}$ & 1.51 & .51 & 2.36 & .71 \\
\hline$\sigma_{\mathrm{L}}$ & .93 & .49 & 1.92 & 1.20 \\
\hline $\mathrm{L}_{\mathrm{CR}}$ & 1.39 & .41 & 2.32 & .72 \\
\hline$\sigma_{\text {LCR }}$ & .84 & .51 & 1.51 & 1.01 \\
\hline $\mathrm{L}_{\mathrm{ERR}}$ & 2.40 & 1.32 & 2.76 & .86 \\
\hline$\sigma_{\text {LEER }}$ & 1.20 & .59 & 1.99 & .81 \\
\hline
\end{tabular}

$\mathrm{L}(\sigma)$. Table 3 gives means and standard deviations for these values. The two subject groups differed signiticantly in mean latency, $t(8)=1.93, p<.05$. and showed a nonsignificant trend to differ in latency variance, $t(8)=1.54, p<.10$. Since reflective children did tend to make the occasional very long response $(>10.12 \mathrm{sec})$, it may be, as we have suggested, that the latency measures in the MFF test, based upon about 12-24 trials, are not highly reliable.

A further breakdown of the latency data for correct and incorrect responding is of some interest. Table 3 also shows mean latencies for correct $\left(\mathrm{L}_{\mathrm{CR}}\right)$ and incorrect (LERR) responses and the mean within-subject standard deviations for both distributions in both subject groups. $t$ tests showed that correct responding was significantly faster than incorrect responding in 8 out of 10 subjects. No further breakdown which would still allow reasonable analysis is possible since the ns associated with the different error categories would be quite small. It may be worth carrying more extensive testing of children. though practical feasibility is something of a problem. to attempt a more detailed verification of speed-accuracy tradeoff models (cf. Pike, McFarland, \& Dalgleish, 1974).

\section{DISCUSSION}

We have been unable to confirm the general value of the impulsive-reflective distinction, since fast responding is not associated with lower recognition accuracy. Latency differences are associated, rather. with changes in response bias.

The differences between the groups in response bias are of some interest. Impulsive children tended to maximize correct "new" responses, while reflective children maximized correct "old" responses. Conceivably, the impulsive group have adopted a more extreme version of the ordinary strategy for picture matching. Jones (1974) argued that children in a picture-recognition cundition might be biased when uncertain toward saying "new" since pictures are particular rather than generic. In practice, a random sample of 5-year-olds was biased toward "old" though this bias was significantly less extreme in the case of picture recognition compared to word recognition (Jones, 1974). The impulsive group may only have pushed this reasonable strategy a little further.

For present purposes, probably the more interesting point is that reflective children tend to adopt rather less extreme biases or, put another way, a rather more cautious response strategy, and that latency is negatively correlated with bias: the more extreme the bias, the lower the speed of responding. This finding is getierally consistent with adult data, where lower latencies do seem to be associated with increased response bias (or higher confidence levels in rating scale experiments, e.g., Jamieson \& Petrusic, 1975; Norman \& Wickelgren, 1969). In other words, latency is related to the distance from the criterion point for "old" or "new."

The relationship between latency and correct/ incorrect responding in the present study is also comparable to adult data. A number of psychophysical studies with adults have shown that errors (or at least nondominant responses) generally have rather longer latencies (e.g., Jamieson \& Petrusic, 1975; Pike et al., 1974). Other work with children (e.g.. Jones, 1975) has turned up similar results. Insofar as Kagan's theory would seem to predict the reverse relationship between latency and correctness of responding from what we observe here, it may be, as we have suggested, that the MFF contounds a number of different factors. Assuming that one possible confound was removed by requiring the subject to make only one choice for any one item, it is then quite conceivable that some children would make more errors in a shorter time on average compared to others who make fewer errors in a longer average time, yet. for each child, his correct responses would be quicker than his incorrect responses. The operational definition of impulsivity-reflectivity would then need to be considerably more complicated than it now is.

In the present case. there is reason to prefer the "impulsive" child's strategy since it leads to the same accuracy in a shorter time. Writers using the MFF, on the other hand, have implicitly valued "reflective" over "impulsive" performance. (It may be worth noticing that terms like "impulsive" and "reflective" are heavily value-laden, and while we would not be inclined to search for "value-free" terms, the substitution of, say, "alert" and "plodding" would give a different impression of which strategy is more highly valued.) The attempt has not been made, for example, to train "reflective" children to make their initial responses more quickly, because it has been assumed that they make more sensitive discrimina- 
tions (e.g., Egland, 1974). Egland (1974) did show that training "impulsive" children can result in longer latencies and fewer total errors on average. However, training strategies may do more than convey the experimenter's wishes to the child in a more explicit manner. The "impulsive" child may not have been taught a more effective way of making perceptual discriminations; rather, he may have been taught that the experimenter did not wish the MFF problem solved in a particular way, that what was really required was the right answer the first time even though the task instructions explicitly allow several choices. The child may move to change his "game plan," but there is no necessary evidence that he has increased discriminative sensitivity.

Possibly the common practice of reporting and analyzing total errors on the MFF has helped to overvalue the reflective child's performance. For example, the "reflective" children in the present study made an average total error of 12.2 on 12 items of the MFF, while the "impulsive" children made a mean total error of 19.8. Put another way, the reflective child was generally correct on his second choice and the impulsive child on the second or third choice. However, the impulsive child took only $5.86 \mathrm{sec}$ on average to make his first choice, compared to $16.18 \mathrm{sec}$ for the reflective child. Similarly, in Egland's (1974) study, the pre- and posttraining scores for $8 \mathrm{MFF}$ items were, respectively, 19.0 errors and 48.6 sec total time compared with 10.4 errors and $125.6 \mathrm{sec}$. The "impulsive" child, when trained to be "reflective," took, in other words, $10 \mathrm{sec}$ longer on average for his first choice, which enabled him to pick the correct items on his second rather than his third choice. Certainly, the child's performance had changed; it had not obviously improved.

We have shown that so-called impulsive and reflective children may not differ in recognition accuracy though they do differ in response bias and in the average speed of response. There is, of course, every reason to study individual differences in speed-accuracy tradeoffs and their relationship to cognitive development. However, such relationships are likely to be rather more complex than they appear at first sight, and there may, in many cases, be no good reason to prefer one child's strategy to another's.

\section{REFERENCES}

Egland, B. Training impulsive children in the use of more efticient scanning techniques. Child Development, 1974, 45. 165.171.

GardNer. R. M., MacFeE, M., \& Krunsky, R. A comparison of binary and rating techniques in the signal detection analysis of recognition memory. Acta Psychologica, 1975, 39, 13-19.

INGLEBY, J. D. The separation of bias and sensitivity in multiple choice tasks. Perception, 1974, 2, 295-305.

Jamieson. D. G., \& Petrusic, W. M. Presentation order effects in duration discrimination. Perception \& Psychophysics, 1975, 17. 197-202.

JONES, B. Response bias in recognition memory for pictures and words by children. Journal of Experimental Psychology. 1974. 103. 1214-1215.

JoNEs. B. Visual facilitation of auditory localization in children: A signal detection analysis. Perception \& Psychophysics, 1975. 17, 217-220.

KAGAN, J. Development studies in reflection and analysis. In A. H. Kidd \& J. H. Rivorie (Eds.), Perceptual development in children. New York: International Universities Press, 1966. (a)

KaGAN, J. Reflection impulsivity: The generality and dynamics of conceptual tempo. Joumal of Abnormal Psychology, 1966, 71, 17-24. (b)

Kagan, J., Rosman, B. L., Day, D., Albert, J., \& Pailips, W. Information processing in the child. Psychological Monographs, 1964. 78(Whole No. 578).

LucE, R. R. Detection and recognition. In R. D. Luce, R. R. Bush, \& E. E. Galanter (Eds.). Handbook of mathematical psychology (Vol. I). New York: Wiley, 1963.

MCNicol. D. A primer of signal detection theory. London: Allen \& Unwin, 1972.

Norman, D. A., \& Wickelgren, W. Strength theory of decision rules and latency in retrieval from short-term memory. Journal of Mathematical Psychology, 1969, 6, 192-208.

PIXE, R. Response latency models for signal detection in audition. Psychological Review, 1973, 80, 53-68.

Pike, R., McFarland, K., \& Dalgleish, L. Speed-accuracy trade-off models for auditory detection with deadlines. Acta Psychologica, 1974, 38, 379.399.

Shepard. R. N., \& Teghtsoonian, M. Retention of information under conditions approaching a steady state. Journal of Experimental Psychology, 1961, 62, 302-309.

Siegel, A. W., Kirasic, K. C., \& Kilburg, R. R. Recognition memory in reflective and impulsive preschool children. Child Development, 1973, 44, 651-656.

(Received for publication June 1975; revision accepted September 18, 1975.) 